\title{
Mycobacterial Infection is Promoted by Neutral Sphingomyelinase 2 Regulating a Signaling Cascade Leading to Activation of $\beta 1$-Integrin
}

\author{
Yuqing Wu Cao Lia Andrea Riehle ${ }^{a}$ Barbara Pollmeier ${ }^{a} \quad$ Erich Gulbins ${ }^{a, b}$ \\ Heike Grassméa \\ aDepartment of Molecular Biology, University of Duisburg-Essen, Essen, Germany, bDepartment of \\ Surgery, University of Cincinnati, Cincinnati, USA
}

\section{Key Words}

Mycobacterium bovis Bacillus Calmette-Guérin (BCG) - Neutral sphingomyelinase 2 - Ceramide - Macrophages • Migration • $\beta 1$-integrin $・$ Rac1 $・$ Granuloma

\begin{abstract}
Background/Aims: Mycobacteria-induced diseases, especially tuberculosis, cause more than 1 million deaths each year, which is higher than any other single bacterial pathogen. Neutral sphingomyelinase 2 (Nsm2) has been implied in many physiological processes and diseases, but the role of Nsm2 in pathogen-host interactions and mycobacterial infections has barely been studied. Methods: We investigated the role of the Nsm2/ceramide system in systemic infection of mice and murine macrophages with Mycobacterium bovis Bacillus Calmette-Guérin $(B C G)$ as a model for mycobacterial infection. For in vitro assays we isolated bone marrowderived macrophages from Wildtype mice or Nsm2-heterozygous and investigated the role of Nsm2 for macrophage migration/clustering as well as the involvement of p38 mitogenactivated protein kinases (p38K), c-Jun N-terminal kinase (JNK), $\beta 1$-integrin and Rac1 activity by Western blot and microscopic studies. For in vivo assays we injected mice intravenously with BCG and analyzed infected tissues for the role of Nsm2-mediated activation of $\beta 1$-integrin in granuloma formation and bacterial burden. Results: Our results reveal that BCG infection of macrophages results in rapid stimulation of Nsm2. Genetic and pharmacological studies demonstrate that Nsm2 stimulates a signaling cascade via p38K and JNK to an activation of surface $\beta 1$-integrin and Rac1 that leads to the formation of granuloma-like macrophages clusters in vitro and granuloma in vivo. Heterozygosity of Nsm2 in macrophages or antibodymediated neutralization of active $\beta 1$-integrin reduced macrophage clusters in vitro and granuloma formation in vivo. Most importantly, Nsm2 heterozygosity or treatment with neutralizing antibodies against $\beta 1$-integrin protected mice from systemic BCG infections and
\end{abstract}




\section{Cellular Physiology Cell Physiol Biochem 2018;51:1815-1829

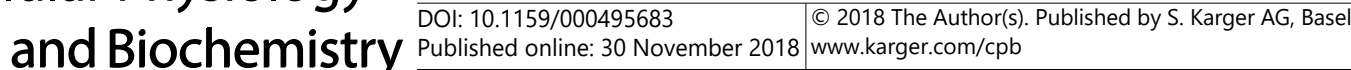 \\ Wu et al.: BCG Induces Granuloma via Neutral Sphingomyelinase and $\beta 1$-Integrin}

chronic infections of the liver and spleen. Conclusion: The findings indicate that the Nsm2/ ceramide system plays an important role in systemic infection of mice with mycobacteria by regulating a signaling cascade via $\mathrm{p} 38 \mathrm{~K}, \mathrm{JNK}, \beta 1$-integrin and Rac1.

(C) 2018 The Author(s)

Published by S. Karger AG, Basel

\section{Introduction}

According to the World Health Organization (WHO), tuberculosis (TB) is one of the leading causes of death from a single infectious agent ranking above HIV/AIDS [1]. Nearly 10 million people worldwide are newly infected with Mycobacterium tuberculosis (M. tuberculosis) each year and approximately 1.6 million die annually as a consequence of this disease; most of the patients have latent TB without clinical symptoms. In all countries, M. tuberculosis is the primary cause of TB. In Africa, tuberculosis is, after AIDS, the deadliest infectious disease, and in Eastern Europe, the incidence of TB is dramatically increasing because of problematic social living conditions and the infection with multi-resistant mycobacteria. These multiresistant pathogens are responsible for the increasing frequency of this disease worldwide [1].

Here, we investigated the role of neutral sphingomyelinase 2 (Nsm2) in the formation of granuloma induced by Mycobacterium bovis Bacillus Calmette-Guérin (BCG) infection in mice. Sphingomyelinases are enzymes characterized by their $\mathrm{pH}$ dependency: acid, neutral, or alkaline. Sphingomyelinases catalyze the breakdown of sphingomyelin into phosphocholine and ceramide, often in response to environmental stress or inflammatory cytokine stimuli $[2,3]$. Nsm2 is a membrane-associated enzyme located in the inner leaflet of the plasma membrane and Golgi membranes; this enzyme releases ceramide in the cytoplasmic leaflet of cellular membranes. This release of ceramide has been reported to occur in various cellular and pathological processes, such as cardiovascular pathophysiologies, neuropathologies, Alzheimer disease, cancer, and bone development [3]. Nsm2 exists in various isoforms. In mammalian cells, Nsm2, a product of the SMPD3 gene, is the main neutral sphingomyelinase responsible for stress-induced generation of ceramide and pathogen-host-interactions [3, 4].

Our previous work has demonstrated that Nsm2 plays an important role in BCG infection, both in macrophages and in vivo [5]. BCG infection of RAW macrophages results in rapid activation of Nsm2 and a massive release of superoxide [5]. Genetic knock-down of Nsm2 in RAW cells prevents excessive superoxide production upon BCG infection. High levels of superoxide suppress autophagy in BCG-infected macrophages. Knock-down of Nsm2 or inhibition of superoxide restores autophagy in macrophages and increases the killing of intracellular bacteria upon BCG infection in vitro. Likewise, autophagy is increased in mice heterozygous for Nsm2, protecting these mice from acute and chronic BCG infections. However, signaling events that mediate the effects of Nsm2 in BCG infections are presently unknown.

Here we report, that Nsm 2 triggers a signaling cascade via $\mathrm{p} 38 \mathrm{~K}, \mathrm{JNK}, \beta 1$-integrin, and Rac1 to induce the formation of macrophage cluster in vitro that are similar to granuloma in vivo. Heterozygosity of the Nsm 2 and antibody-mediated neutralization of active $\beta 1$-integrin reduce the formation of BCG-containing granuloma in the liver and spleen of BCG-infected mice and promote elimination of the pathogen in vivo. Our findings may provide insights into therapeutic possibilities via inhibition of Nsm2 for mycobacterial infections.

\section{Materials and Methods}

Mice, cells, inhibitors

Mice heterozygous for Nsm2 (Nsm2+/; sphingomyelin phosphodiesterase 3 heterozygote; Smpd3) and syngenic Wildtype (Wt) littermates were purchased from the European Mouse Mutant Archive (EMMA; Consiglio Nazionale delle Ricerche, France) and maintained on a $129 \mathrm{sv}$ genetic background. Mice 


\section{Cellular Physiology Cell Physiol Biochem 2018;51:1815-1829

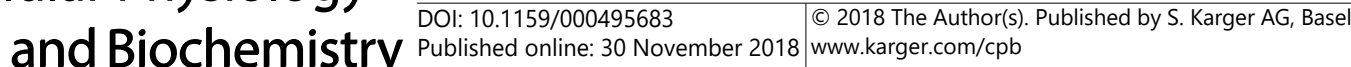 \\ Wu et al.: BCG Induces Granuloma via Neutral Sphingomyelinase and $\beta 1$-Integrin}

were housed in the animal facility of the University of Duisburg-Essen under pathogen-free conditions according to the criteria of the Federation of Laboratory Animal Science. The genotype was verified by polymerase chain reaction (PCR). In vivo infections were approved by the Landesamt für Natur, Umwelt und Verbraucherschutz (LANUV); animal grant G 1312/12 and G 1691/18. We used Nsm2 ${ }^{+/-}$mice in this study and did not employ knock-out mice for the Nsm2, because of the severe phenotype of the complete knockout and ethical reasons. Nsm2 $2^{+-}$mice showed a reduction of $\mathrm{Nsm} 2$-activity of about $30 \%$, but do not develop any obvious spontaneous phenotype and also do not show any severe bone disease.

The in vitro experiments were performed with bone marrow-derived macrophages (BMDMs) obtained from Wt or Nsm2+/- mice. The culture of BMDMs has been previously described in detail [6]. Briefly, mice were sacrificed, and femurs and tibias were flushed with minimum essential medium (MEM; Gibco, Paisley, UK) supplemented with 10\% fetal bovine serum (Gibco), 10 mM HEPES (Roth GmbH, Karlsruhe, Germany; pH 7.4), $2 \mathrm{mM}$ L-glutamine, $1 \mathrm{mM}$ sodium pyruvate, $100 \mu \mathrm{M}$ nonessential amino acids, $100 \mathrm{U} / \mathrm{mL}$ penicillin, and $100 \mu \mathrm{g} / \mathrm{mL}$ streptomycin (Gibco). Isolated cells were passed through a 23-G needle for obtaining single cells, which were cultured for $24 \mathrm{hrs}$ in small tissue-culture flasks. Cells were washed, and $3 \times 10^{4}$ or $1.2 \times 10^{5}$ non-adherent cells were cultured in 24- or 6-well plates in MEM with 20\% L-cell supernatant as a source of macrophage colony-stimulating factor (M-CSF). Fresh MEM/L-cell supernatant medium was applied after 4 days of culture. Macrophages matured within the next 6 days and were used on day 10 of culture.

Inhibitors: In the present study we used the Rac-1 inhibitor NSC23766 (Sigma-Aldrich, Steinheim, Germany) at a concentration of $100 \mu \mathrm{M}$, the p38 MAPK inhibitor SB239063 (Sigma-Aldrich) at $10 \mu \mathrm{M}$ and the JNK inhibitor SP600125 (Sigma-Aldrich) at $10 \mu$ M. Cells were pre-incubated with the inhibitors for $1 \mathrm{hr}$.

\section{Infection experiments}

All in vivo and in vitro infections were performed with green fluorescent protein (GFP)-expressing BCG (GFP-BCG) [7]. The GFP-BCG strain was constructed by transforming BCG with the dual reporter plasmid pSMT3L×EGFP [7]. For infection experiments, bacteria were cultured at $37^{\circ} \mathrm{C}$ in Erlenmeyer flasks with 10 $\mathrm{ml}$ Middlebrook 7H9 Broth with Glycerol (BD Biosciences, Heidelberg, Germany), supplemented with 50 $\mu \mathrm{g} / \mathrm{ml}$ hygromycin B for maintaining GFP-plasmids. Bacteria were used for infection experiments after 5 to 7 days of culture. Bacteria were collected by centrifugation at $880 \times \mathrm{g}$ for $10 \mathrm{~min}$. The bacterial pellet was resuspended in HEPES/saline buffer (H/S) consisting of $130 \mathrm{mM} \mathrm{NaCl}, 1 \mathrm{mM} \mathrm{CaCl}, 0.7 \mathrm{mM} \mathrm{MgCl}, 20 \mathrm{mM}$ HEPES (pH 7.3), $5.4 \mathrm{mM} \mathrm{KCl}$, and $0.8 \mathrm{mM} \mathrm{MgSO}_{4}$ and were vortexed for $5 \mathrm{~min}$. Samples were bath-sonicated for $5 \mathrm{~min}$ at $4^{\circ} \mathrm{C}$ and were passed 10 times through a syringe with a needle $0.8 \mathrm{~mm}$ in diameter. Clumps of bacteria were removed by centrifugation for $2 \mathrm{~min}$ at $220 \times \mathrm{g}$. The supernatant containing single cells of GFP-BCG was carefully collected. The bacterial number was calculated with a $100 \times$ oil lens of an inverted fluorescence microscope (DMIRE2; Leica, Heidelberg, Germany).

For in vitro assays, BMDMs were left uninfected or were infected with GFP-BCG in MEM/10 mM HEPES (pH 7.4) at a bacteria-to-host cell ratio (multiplicity of infection, MOI) of 5:1 to 10:1 for the indicated time. For investigation of macrophage migration/clustering, cells were left untreated or pretreated with $1 \mu \mathrm{g}$ anti31-integrin antibody (rat anti-mouse CD29, clone 9EG7, BD Bioscience) or isotype control (BD Biosciences) $1 \mathrm{hr}$ before infection and then every $5 \mathrm{hrs}$ until $25 \mathrm{hrs}$ during infection. If indicated, $100 \mu \mathrm{M}$ of the Rac1 inhibitor NSC23766 was added $1 \mathrm{hr}$ before infection. In the experiments that analyzed mechanisms of $\beta 1$-integrin activation we added the p38 MAPK inhibitor SB239063 $(10 \mu \mathrm{M})$, the JNK inhibitor SP600125 $(10 \mu \mathrm{M})$, or an equal amount of DMSO $1 \mathrm{hr}$ prior infection to the cells. Synchronous infection conditions and enhanced interactions between bacteria and host cells were achieved by centrifugation $(55 \times \mathrm{g})$ of the bacteria onto the cells for $8 \mathrm{~min}$. The end of the centrifugation was defined as the starting point of infection. The infection was terminated by fixation or lysis, as described below.

For in vivo infections, bacteria were prepared as described above and then pelleted at $2.240 \times \mathrm{g}$ for 10 min. BCG bacteria were resuspended in $0.9 \% \mathrm{NaCl}$, and $1 \times 10^{7} \mathrm{CFU}$ bacteria in $200 \mu \mathrm{l}$ were intravenously injected into mice. Mice were left untreated or treated with anti- $\beta 1$-integrin antibody (9EG7) or isotype control (BD Biosciences), which was intraperitoneally injected at a dose of $30 \mu \mathrm{g}$ total every 2-3 days during infection ( 3 times a week). After the indicated time, the animals were sacrificed and livers and spleens were removed for further processing. 


\section{Cellular Physiology Cell Physiol Biochem 2018;51:1815-1829

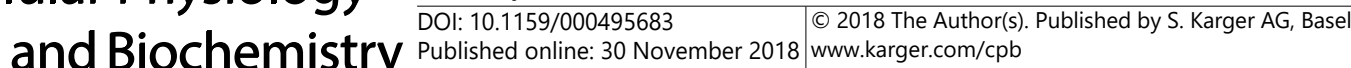 \\ Wu et al.: BCG Induces Granuloma via Neutral Sphingomyelinase and $\beta 1$-Integrin}

\section{Determination of Nsm2 activity}

Nsm2 activity was measured with green fluorescent BODIPY FL $\mathrm{C}_{12}$-sphingomyelin (Thermo Fisher Scientific, Waltham, MA, USA) as a substrate. Briefly, cells were infected or left uninfected, harvested, and lysed in $250 \mathrm{mM}$ sodium acetate (Sigma-Aldrich) and 1\% Nonidet P-40 (pH 7.3; Sigma-Aldrich) for 5 min on ice. Cells were further disrupted by sonication for $10 \mathrm{~min}$ in a bath sonicator at $4^{\circ} \mathrm{C}$ (Bandelin Electronic, Berlin, Germany). The protein concentration was measured by Bradford protein assay (BioRad, München, Germany): $20 \mu \mathrm{g}$ of protein in $20 \mu \mathrm{L}$ lysis buffer was added to $250 \mathrm{mM}$ sodium acetate (pH 7.3) containing 100 pmol BODIPY $\mathrm{FL} \mathrm{C}_{12}$-sphingomyelin. The samples were incubated at $37^{\circ} \mathrm{C}$ for $1 \mathrm{hr}$. The reaction was stopped by the addition of $1 \mathrm{~mL}$ chloroform:methanol (2:1, v/v), followed by centrifugation for $5 \mathrm{~min}$ at $10.510 \times \mathrm{g}$. The lower phase was dried in a SpeedVac Concentrator (Thermo Fisher Scientific) and resuspended in $20 \mu \mathrm{L}$ of chloroform:methanol $(2: 1, \mathrm{v} / \mathrm{v})$. The samples were spotted on a 20-cm thin-layer chromatography (TLC) plate (Merck, Darmstadt, Germany) in 3- $\mu$ L steps. After all spots were dried, the samples were separated with chloroform:methanol (80:20, v/v), scanned with a Typhoon FLA 9500 laser scanner (GE Healthcare Life Sciences, Freiburg, Germany), and analyzed with ImageQuant software (GE Healthcare Life Sciences).

\section{Western blots and pull-down assay}

BMDMs were left uninfected or were infected for the indicated time, washed in cold $\mathrm{H} / \mathrm{S}$ buffer, and lysed for $5 \mathrm{~min}$ on ice in $125 \mathrm{mM} \mathrm{NaCl}, 25 \mathrm{mM}$ Tris- $\mathrm{HCl}$ (pH 7.4), $10 \mathrm{mM}$ ethylenediaminetetraacetic acid (EDTA), $10 \mathrm{mM}$ sodium pyrophosphate, and 3\% NP-40 supplemented with $10 \mu \mathrm{g} / \mathrm{mL}$ aprotinin/leupeptin (A/L). Non-adherent cells in the supernatant were collected and centrifuged at $300 \times \mathrm{g}$ for $5 \mathrm{~min}$. Adherent cells were lysed in lysis buffer as described above. Lysates and pellets were combined, and insoluble material was pelleted by centrifugation for $10 \mathrm{~min}$ at $10.510 \times \mathrm{g}$. The supernatants were added to $5 \times$ sodium dodecyl sulfate (SDS) sample buffer, samples were boiled for $5 \mathrm{~min}$ and separated by $8.5 \%$ to $12.5 \%$ SDS polyacrylamide gel electrophoresis (SDS-PAGE). Samples were blotted on nitrocellulose membranes (Protran Premium $0.2 \mu \mathrm{m}$, GE Healthcare) for $2 \mathrm{hrs}$ at $4^{\circ} \mathrm{C}(80 \mathrm{~V})$. For detection of proteins, blots were washed with PBS and blocked for $1 \mathrm{hr}$ at room temperature in Starting Block Tris-buffered saline (TBS) buffer (Thermo Fisher Scientific). After two additional washes in PBS, specific primary antibodies against phosphorylated p38 or phosphorylated JNK (Cell Signaling Technology, Danvers, MA, USA) were incubated overnight at $4^{\circ} \mathrm{C}$ in blocking buffer at 1:1000 dilution. After 6 washes in TBS/Tween, blots were incubated for $1 \mathrm{hr}$ at room temperature in TBS/Tween containing 10\% blocking buffer with alkaline phosphatase (AP)-conjugated secondary antibodies (Santa Cruz Biotechnology INC, Heidelberg, Germany). Samples were washed extensively and developed with CDP-Star substrate (Perkin Elmer, Rodgau, Germany).

Rac1 activity was detected with an Activation Assay Combo Biochem Kit (Cytoskeleton Inc., Denver, USA) according to the manufacturer's instructions. Briefly, cells were infected and lysed in $50 \mathrm{mM}$ Tris (pH 7.5), $10 \mathrm{mM} \mathrm{MgCl}{ }_{2}, 0.5 \mathrm{M} \mathrm{NaCl}$, and $2 \%$ Igepal. Equivalent amounts of protein were added to a predetermined concentration of p21-activated protein kinase (PAK) binding domain (PBD) (PAK-PBD) beads and incubated at $4^{\circ} \mathrm{C}$ on a rotator for $1 \mathrm{hr}$. Beads were washed with a buffer consisting of $25 \mathrm{mM}$ Tris (pH 7.5), $30 \mathrm{mM}$ $\mathrm{MgCl}_{2}$, and $40 \mathrm{mM} \mathrm{NaCl}$. Finally, $20 \mu \mathrm{L}$ of SDS Laemmli sample buffer were added to each sample. Samples were centrifuged and the supernatants were separated on a 10\% SDS-PAGE gel and transferred to a nitrocellulose membrane. Blots were incubated with monoclonal anti-Rac1 antibodies (ARC03; antibodies from Cytoskeleton Inc.) according to the vendor's instructions and were developed as described above.

\section{B1-integrin immunoprecipitation}

To demonstrate activation of $\beta 1$-integrin on the surface of BMDMs, we infected cells for various time periods, removed the medium, and incubated macrophages on ice for $30 \mathrm{~min}$ with $1 \mu \mathrm{g}$ of anti- $\beta 1$-integrin antibodies, clone 9EG7. The samples were washed extensively and lysed in $125 \mathrm{mM} \mathrm{NaCl}, 25 \mathrm{mM}$ Tris $\mathrm{HCl}$ (pH 7.4), $10 \mathrm{mM}$ EDTA, $10 \mathrm{mM}$ sodium pyrophosphate, $3 \%$ Nonidet P-40, and $10 \mu \mathrm{g} / \mathrm{ml} \mathrm{A} / \mathrm{L}$ for $5 \mathrm{~min}$ at $4^{\circ} \mathrm{C}$. They were then centrifuged at $10.510 \times \mathrm{g}$. The immunocomplexes were immobilized with protein A/G agarose (Santa Cruz Biotechnology, Heidelberg, Germany) for 45 min, washed 6 times in lysis buffer, resuspended in $1 \mathrm{xSDS}$ Laemmli Sample buffer, and boiled for $5 \mathrm{~min}$ at $95^{\circ} \mathrm{C}$. Proteins were separated on 8.5\% SDS-PAGE gels, blotted with $\beta 1$-integrin antibody clone MB1.2 (Merck Chemicals GmbH, Darmstadt, Germany) and developed with an AP-coupled secondary antibody and a chemiluminescence system (Thermo Fisher Scientific).

\section{KARGER}




\section{Cellular Physiology Cell Physiol Biochem 2018;51:1815-1829

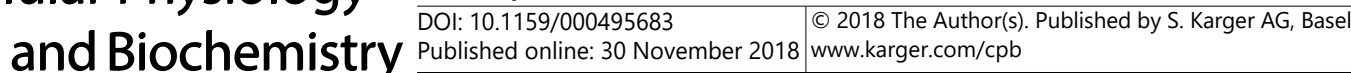 \\ Wu et al.: BCG Induces Granuloma via Neutral Sphingomyelinase and $\beta 1$-Integrin}

\section{Histopathologic assessment}

Mice were sacrificed and livers and spleens were removed, embedded in Tissue-Tec (Sakura Finetek USA, Torrance, CA, USA), and shock-frozen in liquid nitrogen. Sections 6 - $\mu \mathrm{m}$ thick were cut with a cryotome (CM1850 UV, Leica Microsystems). For staining, sections were thawed and fixed in ice-cold acetone for 10 min. For fluorescent visualization of mycobacteria, sections were washed in PBS and were stained with the Truant TB Fluorescent Stain Kit (BD Difco, Becton Dickinson, Franklin Lakes, NJ, USA) following the manufacturer's instructions. Bacterial distribution was analyzed with an inverted fluorescence microscope or with a confocal microscope (DMIRE2; Leica).

For hematoxylin and eosin (H\&E) staining, liver and spleen sections were prepared as above and stained for 20 min with Mayer's hemalaun solution (T865.1; Roth). Samples were washed in water for 15 min, stained for an additional 2 min with 1\% eosin solution, washed with water, dehydrated in ethanol, embedded in Eukitt mounting medium (Sigma-Aldrich), and analyzed on a Leica DMIRE2 microscope (Leica). Granuloma formation was assessed with a $20 \times$ lens and an inverted fluorescence microscope.

For Oil Red 0 staining, liver sections were fixed with 4\% PFA for $15 \mathrm{~min}$. Sections were washed, incubated in $85 \%$ propylene glycol for $2 \mathrm{~min}$, stained with $0.5 \%$ Oil red 0 (Abcam, Cambridge, UK) for $1 \mathrm{hr}$ at room temperature, differentiated in $85 \%$ propylene glycol for $1 \mathrm{~min}$, counterstained with Mayer's hemalaun to stain nuclei for $30 \mathrm{sec}$ and imaged by Leica DMIRE2 microscope with a 100×objective lens.

\section{Quantification of bacterial numbers}

To count BCG colony forming units (CFUs) in tissue, we removed the liver and spleen from infected mice, added $5 \mathrm{mg} / \mathrm{mL}$ saponin (Serva Electrophoresis GmbH, Heidelberg, Germany) in H/S, and homogenized the tissues in a loose Dounce homogenizer (Braun, Kronberg, Germany). The homogenates were incubated for $30 \mathrm{~min}$ at $37^{\circ} \mathrm{C}$ in a thermomixer for the release of intracellular bacteria. Samples were centrifuged for $2 \mathrm{~min}$ at 220×g; the supernatant was diluted in PBS and plated on Middlebrook 7H10 agar plates enriched with oleic acid (OADC; BD Biosciences). For CFU assays from macrophages, infected cells were washed once with MEM/10 mM HEPES ( $\mathrm{pH} 7.4$ ) after various infection times to remove non-adherent bacteria and were then lysed in $3 \mathrm{mg} / \mathrm{mL}$ saponin for $30 \mathrm{~min}$ at $37^{\circ} \mathrm{C}$. We plated $100-\mu \mathrm{L}$ aliquots and counted bacterial CFUs after the plates had been incubated for approximately 2 weeks in a humidified $37^{\circ} \mathrm{C}$ atmosphere.

\section{Statistical analysis}

Data are expressed as arithmetic means \pm standard deviation (SD). All data were tested for normal distribution with the David-Pearson-Stephens test. Statistical analysis was performed with Student's $t$-test for single comparisons and with analysis of variance (ANOVA) for multiple comparisons. Statistical significance was set at the level of $p \leq 0.05$. All data were obtained from independent measurements. The GraphPad Prism statistical software program (GraphPad Software, La Jolla, CA, USA) was used for analysis.

\section{Results}

Nsm2 activates a signaling cascade from $p 38 K$ and JNK via $\beta 1$-integrin to Rac1 upon infection with $B C G$

We have previously shown that BCG infection activates Nsm2 and that heterozygosity of the Nsm2 reduces formation of granuloma and the number of BCG in the liver and spleen of BCG-infected mice in vivo [5]. However, signaling molecules that link Nsm2 with the formation of granuloma and BCG infections are unknown.

To identify signaling events that mediate the effects of Nsm2 activation, we investigated the role of $\beta 1$-integrin activation in BMDMs upon BCG infection, because $\beta 1$-integrin has been previously shown to be regulated by ceramide [8] and to be involved in the formation of granuloma [9]. First, we confirmed activation of Nsm2 upon infection of Wildtype (Wt) macrophages with BCG (Fig. 1A). Nsm2-heterozygous ( $\mathrm{Nsm} 2^{+/}$) cells did not respond to infection with an activation of Nsm with BCG (Fig. 1A). Next, we measured $\beta 1$-integrin activation by immunoprecipitation of active surface $\beta 1$-integrin at several time points after BCG infection (Fig. 1B, C). We detected $\beta 1$-integrin activation as early as 30 min after BCG 


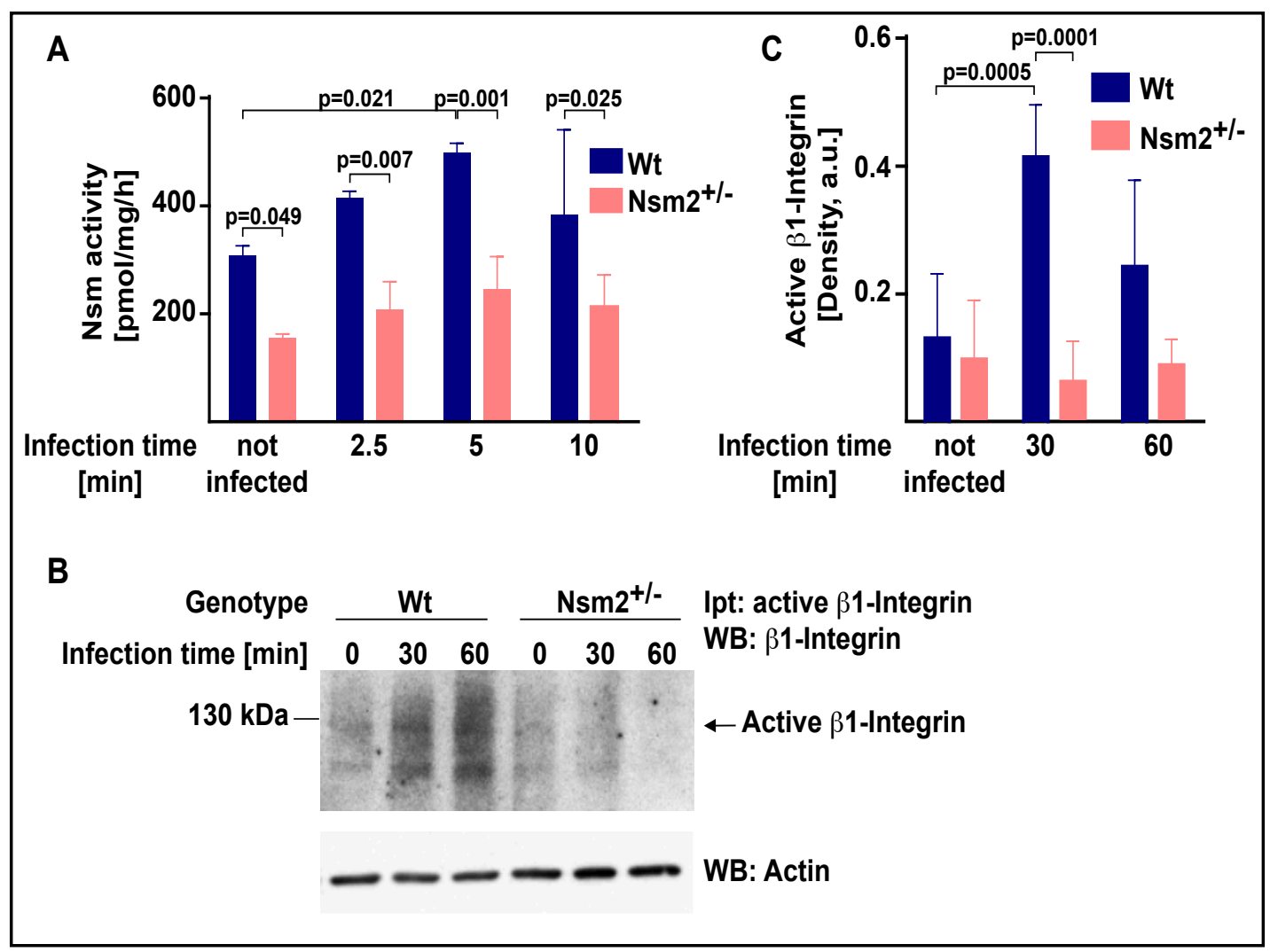

Fig. 1. Infection of macrophages with Bacillus Calmette-Guérin activates neutral sphingomyelinase and $\beta 1$-Integrin. (A) Bone marrow-derived macrophages (BMDMs) from Wildtype (Wt) and neutral sphingomyelinase 2 heterozygous $\left(\mathrm{Nsm}^{+/}\right.$) mice were infected with Bacillus Calmette-Guérin (BCG) for the indicated time, lysed, and the activity of neutral sphingomyelinase (Nsm) was determined by the consumption of BODIPY FL $\mathrm{C}_{12}$-sphingomyelin. Samples were extracted and separated on thin-layer chromatography (TLC) plates, which were scanned with a Typhoon laser scanner. Shown is the mean \pm standard deviation (SD) of the Nsm activity of 3 independent experiments, the exact $\mathrm{p}$ is given as determined by ANOVA followed by Bonferroni's multiple comparisons test. (B) Active $\beta 1$-integrin expression on Wt and $\mathrm{Nsm}^{+/-} \mathrm{BMDMs}$ was determined after BCG infection for 30 or 60 min by immunoprecipitation (Ipt). Western blots (WB) were developed with anti- $\beta 1$-integrin-antibodies. Shown is a representative western blot from 4 independent experiments. (C) Quantitative analysis of activation of $\beta 1$-integrin by immunoprecipitation. Shown is the mean $\pm S D, n=4$, of the density of the western blots as determined by ImageJ, given in arbitrary units (a.u.). P values were determined by ANOVA followed by Bonferroni's multiple comparisons test.

infection in Wt BMDMs. In contrast, $\beta 1$-integrin activation was almost completely absent from Nsm2 $2^{+/-}$BMDMs after BCG infection (Fig. 1B, C).

To identify further signaling events that link Nsm2 activation with granuloma formation and pathogen control after infection of macrophages with BCG and to order these events in relation to $\beta 1$-integrin stimulation, we investigated whether $\mathrm{p} 38$ mitogen-activated protein kinases (p38K) and c-Jun N-terminal kinase (JNK) signaling is involved in BCG-induced macrophage clustering and $\beta 1$-integrin activation in macrophages. We first determined the phosphorylation of $\mathrm{p} 38 \mathrm{~K}$ and JNK at various time points after infection (Fig. 2A-C). The results indicate a marked phosphorylation of p38K and JNK in Wt BMDMs. The levels of phosphorylation of p38K (p-p38) and JNK (pJNK) were much lower in Nsm2 ${ }^{+/-}$BMDMs than in Wt BMDMs upon BCG infection (Fig. 2A-C). Measurement of phosphorylation of Extracellular Regulated Kinase (pERK) showed no difference between Wt and Nsm2 ${ }^{+/-}$BMDMs upon BCG infection (not shown). Protein levels of p38K and JNK did not change after BCG infections indicating a true activation of the two kinases by BCG (not shown).

\section{KARGER}




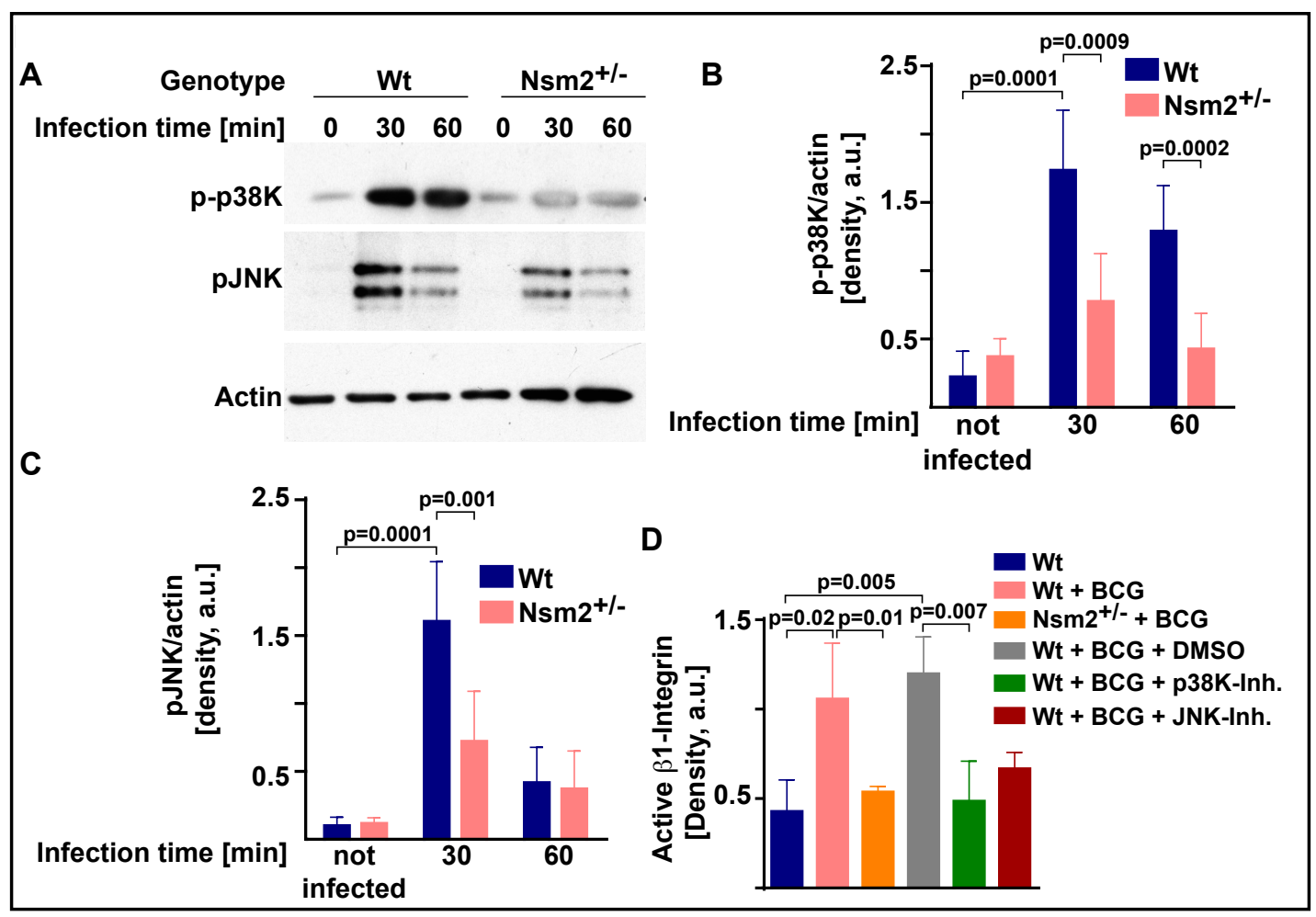

Fig. 2. Bacillus Calmette-Guérin infection activates $\beta 1$-Integrin via neutral sphingomyelinase 2 , $\mathrm{p} 38 \mathrm{~K}$ and JNK. (A) Bone marrow-derived macrophages (BMDMs) from Wildtype (Wt) and neutral sphingomyelinase2 heterozygous $\left(\mathrm{Nsm}^{+/-}\right.$) mice were infected with BCG for the indicated periods of time and were then subjected to Western blot analysis using antibodies of p-p38, p-JNK, and $\beta$-actin. The western blot is representative of 4 independent experiments. Panels (B) and (C) display the quantitation of the phosphorylation of $\mathrm{p} 38 \mathrm{~K}$ and JNK performed using ImageJ. The density of the western blot signals was measured and is given in arbitrary units (a.u.). Activation of p38K and JNK upon infection with BCG depends on expression of Nsm2. Displayed is the mean \pm SD of four experiments, p-values were calculated by ANOVA followed by Bonferroni's multiple comparisons test. (D) The activation status of $\beta 1$-integrin in macrophages after Nsm2 infection was determined by immunoprecipitation. To test whether Nsm2 dependent activation of p38K and JNK and $\beta 1$ integrin activation are connected, $\mathrm{Nsm} 2^{+/-}$or Wt BMDMs were treated for $1 \mathrm{~h}$ prior infection with the p38 MAPK inhibitor SB239063 (10 $\mu \mathrm{M})$, or the JNK inhibitor SP600125 (10 $\mu \mathrm{M})$ and infected for $30 \mathrm{~min}$. Shown is the mean $\pm \mathrm{SD}, \mathrm{n}=4$, of the density of the western blots as determined by ImageJ, given in arbitrary units (a.u.). P values were determined by ANOVA followed by Bonferroni's multiple comparisons test.

To test whether Nsm2-dependent activation of $\mathrm{p} 38 \mathrm{~K} / \mathrm{JNK}$ and $\beta 1$-integrin are connected, we pretreated $\mathrm{Wt}$ macrophages for $1 \mathrm{hr}$ with anti- $\beta 1$-integrin antibodies and then infected them with BCG for various time periods. However, blocking active $\beta 1$-integrin did not change the phosphorylation level of either p38 or JNK (data not shown). In contrast, pretreatment of macrophages with specific inhibitors of JNK (SP600125) or p38K (SB203580) for $1 \mathrm{hr}$ prior infection with BCG for 30 min prevented BCG-induced $\beta 1$-integrin activation (Fig. 2D). The solvent dimethyl sulfoxide (DMSO) was without effect on $\beta 1$-integrin activation by BCG. These findings indicate that $\mathrm{p} 38 \mathrm{~K}$ and JNK are upstream of $\beta 1$-integrin activation.

Previous studies have linked active $\beta 1$-integrin with the stimulation of small G-proteins that are involved in cell migration and the attraction of inflammatory cells to pathogens [10, 11]. Our study demonstrated a marked activation of Rac 1 as early as $30 \mathrm{~min}$ after infection of Wt macrophages, but not in Nsm2+/- BMDMs (Fig. 3A). Neutralization of active $\beta 1$-integrin by incubation of macrophages with anti-active $\beta 1$-integrin antibodies reduced Rac1 activity (Fig. 3B). 


\section{Cellular Physiology Cell Physiol Biochem 2018;51:1815-1829

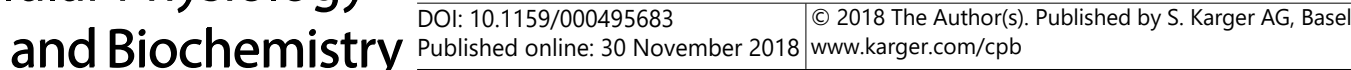 \\ Wu et al.: BCG Induces Granuloma via Neutral Sphingomyelinase and $\beta 1$-Integrin}

Fig. 3. Neutral sphingomyelinase regulates activation of Rac1 via $\beta 1$-integrin after infection with Bacillus Calmette-Guérin. (A) Bone marrowderived macrophages (BMDMs) from Wildtype (Wt) and neutral sphingomyelinase 2 heterozygous $\left(\mathrm{Nsm}^{+/-}\right)$mice were infected with BCG for the indicated time, lysed and Rac1 activation was determined by pull-down assays. Shown are representative results from 3 independent studies. (B) The connection from Rac1 to integrin activation was determined by pretreating Wt BMDMs with anti- $\beta 1$-integrin antibody 9EG7 starting $1 \mathrm{hr}$ before infection with BCG. Untreated or antibody treated macrophages were then left uninfected or infected with BCG for the indicated time points. Rac1 activity was detected with a Rac1 pull-down assay. Shown are representative results from 4 independent studies.

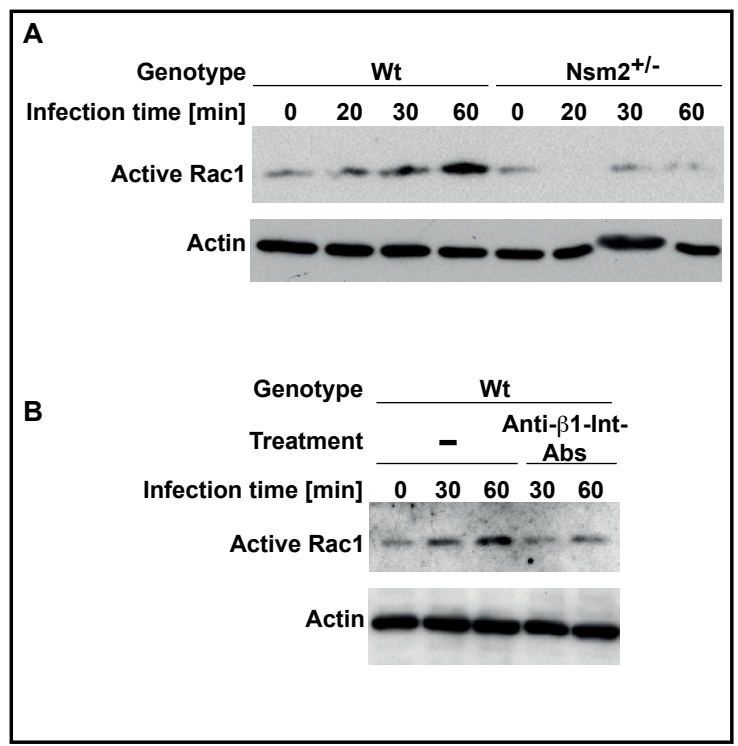

Nsm2-dependent activation of $p 38 K$ and JNK via $\beta 1$-integrin to Rac1 mediates granuloma formation

To investigate the significance of the signaling pathway from Nsm2 to $\beta 1$-integrin and Rac1, we investigated the role of these molecules in hallmarks of the infection, i.e., granuloma formation and persistence of the pathogen. We have previously shown that Nsm 2 is important for the formation of granuloma and the survival of BCG in vivo [5]. Heterozygosity of Nsm2 reduced granuloma formation and survival of the pathogen in the liver and spleen in vivo [5]. Here, we extended and confirmed these data and investigated granuloma formation up to 12 weeks. Wt and $\mathrm{Nsm} 2^{+/-}$mice were intravenously infected with $1 \times 10^{7}$ bacteria for various periods of time to discriminate between acute and chronic infection. While we detected no differences in granuloma formation between Wt and Nsm2-heterozygous mice in the liver 1 week after infection (Fig. 4A), we observed a marked difference of granuloma between $\mathrm{Wt}$ and Nsm2-heterozygous mice starting with 3 weeks up to 6 weeks post-infection (Fig. 4A). Granulomas were nearly completely absent from both Wt and $\mathrm{Nsm}^{+/-}$livers at 12 weeks after infection (Fig. 4A). Furthermore, in contrast to $\mathrm{Nsm}^{+/-}$mice, the granuloma in livers of Wt mice after 3 weeks of infection showed much more accumulation of lipid droplets that are considered a typical feature of mycobacterial granuloma [12] (Fig. 4B).

To test whether $\beta 1$-integrin, which functions downstream of Nsm2, is involved in granuloma formation and elimination of the pathogen, we pretreated mice with either anti$\beta 1$-integrin antibody or an isotype control antibody 3 times per week during infection or left the mice untreated and infected them with BCG for 3 weeks. Animals injected with active $\beta 1$ integrin exhibited fewer granulomas than control animals and these granulomas were less organized and devoid of a large center (Fig. 4C, D). Red Oil 0 staining revealed that anti- $\beta 1$ integrin antibody treated mice had dramatically reduced lipid droplets accumulation after 3 weeks infection, compared to isotype control antibody treated mice, and Wt mice (Fig. 4E).

We also assessed the function of active $\beta 1$-integrin for the persistence of BCG upon infection in vivo. Our findings show that 3 weeks after BCG infection bacterial numbers in livers and spleens were $40 \%$ to $50 \%$ lower in animals treated with neutralizing anti- $\beta 1$ integrin antibodies than in Wt mice or mice treated with isotype control antibodies (Fig. 5A, B).

These findings indicate that the Nsm2 determines the formation of granulomas and the persistence of BCG in vivo via an activation of active $\beta 1$-integrin. 


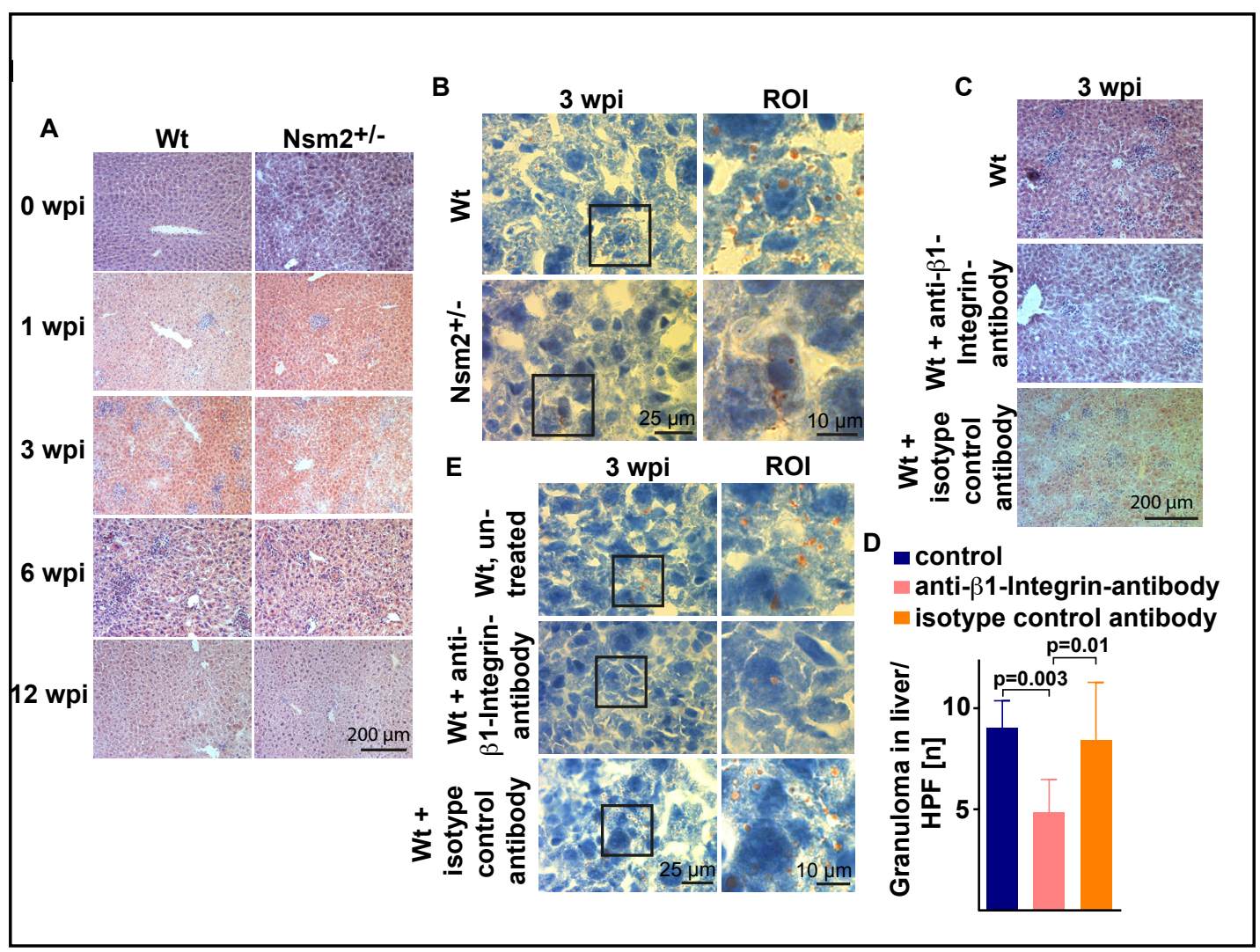

Fig. 4. Neutral sphingomyelinase induces granuloma formation in mice in vivo via $\beta 1$-integrin. (A) Wildtype (Wt) or neutral sphingomyelinase 2 heterozygous $\left(\mathrm{Nsm}^{+/-}\right)$mice wereleft uninfected $(0$ weeks post-infection, wpi) or infected with $1 \times 10^{7}$ Bacillus Calmette-Guérin bacteria per mouse for 1 to 12 weeks post-infection (1 -12 wpi). Cryosections of liver tissue were stained with hematoxylin and eosin (H\&E) and analyzed by light microscopy with a $20 \times$ lens. Shown are pictures representative of four independent experiments. The scale bar represents $200 \mu \mathrm{m}$.(B) Lipid drops in liver sections from Wt and $\mathrm{Nsm} 2^{+/-}$mice 3 weeks after infection were visualized with Oil Red staining and analyzed by light microscopy with a $100 \times$ lens. Shown are pictures representative of four independent experiments. The scale bar in the overview represents $25 \mu \mathrm{m}$ and in the regions of interest (ROI) $10 \mu \mathrm{m}$. (C-E) Wildtype (Wt) mice were infected with BCG for 3 weeks (3 wpi) with or without treatment with anti- $\beta 1$-integrin antibody or its isotype control 3 times per week. Panel (C) displays representative hematoxylin and eosin (H\&E) from liver section from 3 independent experiments. (D) The number of granulomas in liver tissue was quantified by counting granulomas in a total of 30 serial sections (10 sections per mouse tissue) by H\&E staining. Given is the number per high power field (HPF) using a $20 \times$ lens. Shown is the mean \pm SD,$n=3$, ANOVA. (E) Accumulation of lipid drops in liver sections was visualized with Oil Red stain and analyzed by light microscopy with a 100×lens. Shown are pictures representative of four independent experiments. Scale bar in the overviews represents $25 \mu \mathrm{m}$ and in the regions of interest (ROI) $10 \mu \mathrm{m}$. 


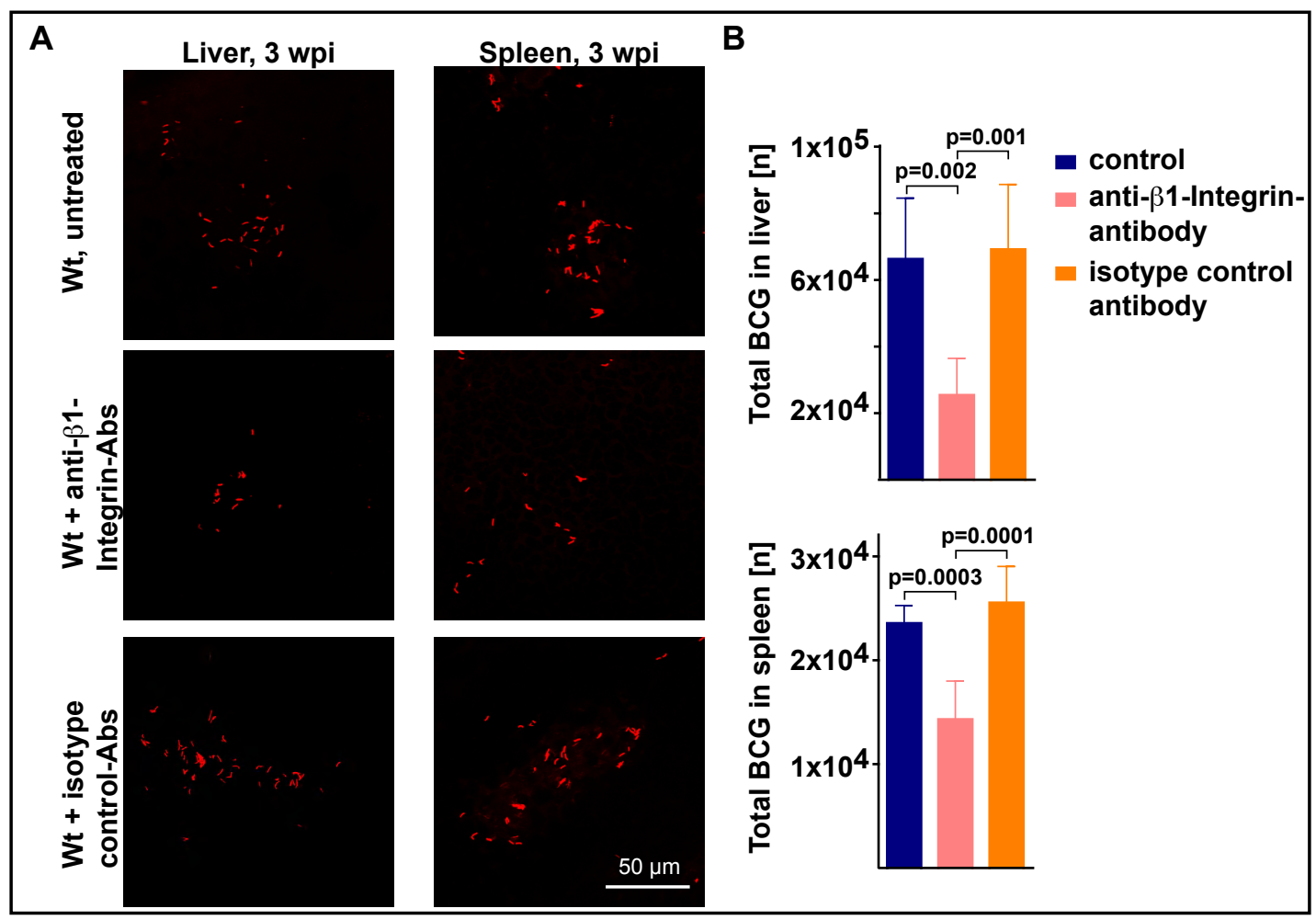

Fig. 5. Neutralization of $\beta 1$-integrin reduces in vivo infection with Bacillus Calmette-Guérin. (A) Wildtype $(\mathrm{Wt})$ mice were infected with BCG for 3 weeks ( 3 wpi) with or without pretreatment with anti- $\beta 1$-integrin antibody or its isotype control 3 times per week during infection. Liver and spleens were removed and stained with Truant dye for visualization of mycobacteria and analyzed by fluorescence microscopy using a $63 x$ lens. Shown are pictures representative of 6 independent experiments. Scale bar is $50 \mu \mathrm{m}$. (B). The total number of BCG in tissues homogenates was determined by colony-forming unit (CFU) assays. Livers or spleen were homogenized, the extracts were lysed by saponin and CFUs of BCG were determined on plates. Experiments were repeated at least 6 times. Shown is the mean \pm SD of the absolute number (n) of BCG in the liver and spleen. The exact $\mathrm{p}$ is given as determined by ANOVA followed by Tukey's multiple comparisons test.

To further support the notion that Nsm2 regulates granuloma formation and BCG survival via active $\beta 1$-integrin and Rac1, we used an in vitro model that mimics some aspects of granuloma formation in vivo. Infection of cultured macrophages with BCG induces migration and clustering of macrophages looking similar to granuloma (Fig. 6A). We used hematoxylin and eosin (H\&E) staining to investigate macrophage clustering 10 to $30 \mathrm{hrs}$ after infection of BMDMs from Wt and Nsm2-heterozygous mice (Fig. 6A and B). In Wt macrophages, BCG triggered the formation of large macrophage clusters, which were almost absent in Nsm2heterozygous cells. Macrophage clusters in these cultures were much smaller and also the number was reduced (Fig. 6A, B).

To investigate the function of active $\beta 1$-integrin for clustering of BMDMs, we infected BMDMs for $25 \mathrm{hrs}$ and neutralized $\beta 1$-integrin on cells by treating them with anti- $\beta 1$ integrin antibodies every 5 hrs during infection (for a total of 5 times). The neutralization of $\beta 1$-integrin substantially reduced the size and number of macrophage clusters formed by $\mathrm{Wt}$ macrophages, whereas neutralizing anti- $\beta 1$-integrin antibodies had no effect on macrophage clusters formed by Nsm2+/- BMDMs (Fig. 6C).

To define the function of Rac1 activation in the formation for macrophage clustering, we determined macrophage clustering after pretreating BMDMs with an inhibitor of Rac1 (NSC23766) for $1 \mathrm{hr}$ before infection with BCG (Fig. 6D). Rac1 inhibition markedly reduced formation of macrophage clusters.

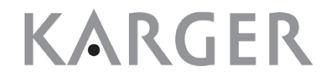




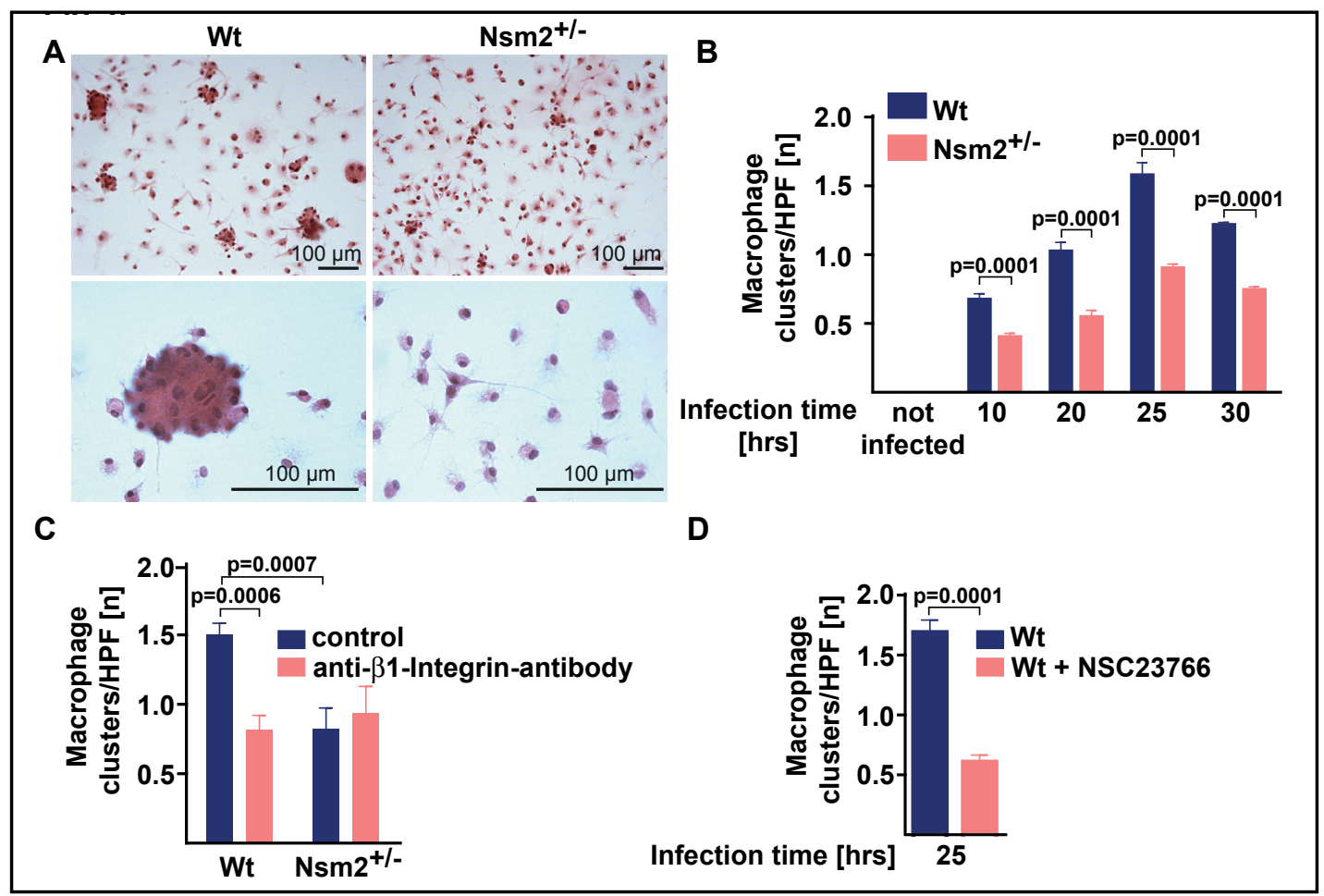

Fig. 6. Generation of macrophage clusters upon BCG infection is controlled by Nsm2-dependent activation of $\beta 1$-integrin and Rac1. (A) Wildtype (Wt) or $\mathrm{Nsm}^{+/-}$BMDMs were infected for $25 \mathrm{hrs}$, fixed, and stained with hematoxylin and eosin (H\&E). Granulomas were examined with a $40 \times$ lens by light microscopy. The scale bar is $100 \mu \mathrm{m}$. The pictures represent the results of 4 independent experiments. (B) Macrophage cluster forming granuloma-like structures of Wildtype (Wt) or $\mathrm{Nsm}^{+/}$BMDMs were quantified after BCG infection for the indicated time by counting clusters of at least 5 macrophages in 100 fields per microscopic study using a 40x lens. (C, D) Wildtype (Wt) BMDMs were infected with BCG for $25 \mathrm{hrs}$. (C) $\beta 1$-integrin was neutralized by pretreatment with anti- $\beta 1$-integrin antibody 9EG7 $1 \mathrm{hr}$ before infection and then every $5 \mathrm{hrs}$ until 25 hrs during infection with BCG. (D) Rac1 was inhibited using the inhibitor NSC23766 starting $1 \mathrm{hr}$ before infection. Given is the number (n) of macrophage clusters per high power field (HPF). Shown is the mean \pm SD of 4 (A-C) or 3 (D) independent experiments (thus a total of $300-400$ microscopy fields were studied). The exact $\mathrm{p}$ is given as determined by ANOVA followed by Bonferroni's multiple comparisons test.

\section{Discussion}

Our data show that BCG infection activates Nsm2 in macrophages, thereby triggering the activation of the $\mathrm{p} 38 \mathrm{~K} / \mathrm{JNK}$ pathway followed by stimulation of surface $\beta 1$-integrin, which in turn induces an activation of Rac1 and finally granuloma formation and persistence of the pathogen (Fig. 7). Heterozygosity of Nsm2 or neutralization of active $\beta 1$-integrin by antibodies disrupts the formation of granulomas and reduces the bacterial burden in infected hosts indicating the significance of this signaling cascade for BCG infection (Fig. 7).

These data might support the notion that granulomas are a secure reservoir for mycobacteria and a favorable niche for bacteria in the infected host, which is prevented in mice heterozygous for the Nsm 2 or upon application of anti- $\beta 1$-integrin antibodies. In this scenario, the initial infection would be similar in Wildtype and Nsm2-heterozygous mice, but the lack of granuloma formation in Nsm2-heterozygous mice allows more efficient elimination of the bacteria. Since our data show no difference in the number of small granuloma one week after infection, we favor this scenario, although our data do not entirely exclude that reduced granuloma formation in Nsm2-heterozygous mice is secondary to an improved killing in mice heterozygous for the Nsm2.

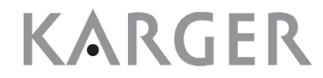




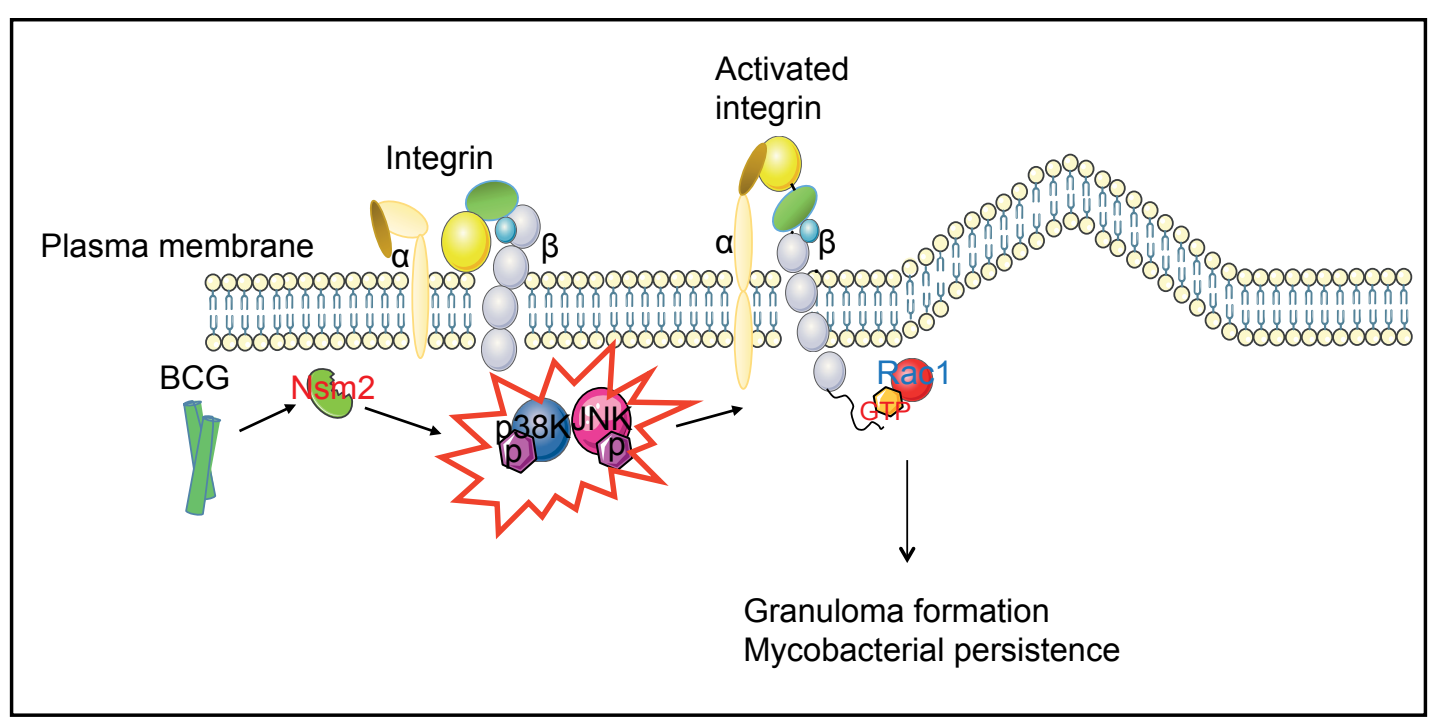

Fig. 7. Scenario of $\beta 1$-integrin regulated granuloma formation upon BCG infection. BCG infection activates Nsm2 in macrophages, resulting in p38K/JNK activation followed by stimulation of surface $\beta 1$-integrin and Rac1 activation, thus leading to migration of macrophages and the formation of multiple granulomas. The pathway controls granuloma formation and mycobacterial persistence in vivo.

Previous studies of human TB granulomas have shown that active $\beta 1$-integrin controls granuloma formation by mediating the cell fusion machinery [9]. Incubating macrophages with anti- $\beta 1$-integrin antibodies completely abolishes mycobacterial lipomannan-induced cell aggregation [9]. These findings suggest that $\beta 1$-integrin is directly involved in granuloma formation. In vivo, anti- $\beta 1$-integrin antibodies may not only affect macrophages, which are in the focus of the present study, but may also prevent the recruitment of other cells that are present in the granuloma, in particular, dendritic cells and T lymphocytes. Inhibition of granuloma formation by affecting the recruitment of a variety of cells, including macrophages and $\mathrm{T}$ lymphocytes, may allow rapid elimination of single BCG, while in Wildtype mice the formation of granuloma seems to promote persistence of the bacteria. Since inhibition of $\beta 1$ integrin by injection of neutralizing antibodies promotes elimination of BCG, the inhibition of $\beta 1$-integrin seems to prevent recruitment of macrophages and Tymphocytes to granuloma, but not the function of these cells against single BCG.

Integrins are widespread adhesion receptors and mediate migration. They are also mechanosensors and mediate the response to mechanical forces across cell membranes [13, 14]. Among these, $\beta 1$-integrin is one of the most common integrins associated with bacterial adhesion and actin cytoskeleton rearrangements [13]. In addition, $\beta 1$-integrin leads to the invasion of mycobacteria into macrophages, their persistence in these cells, and their spread into other macrophages [15]. It has been previously reported that the fusion of mycobacteriainduced macrophages and the generation of in vitro granulomas are in part dependent on $\beta 1$-integrin $[9,16]$. However, the mechanisms behind $\beta 1$-integrin activation and its exact role in infection remained unknown. Our work, for the first time, indicates that activation of $\beta 1$-integrin is regulated by the Nsm2/ceramide system via $\mathrm{p} 38 \mathrm{~K}$ and JNK, as indicated by the use of pharmacological blockers. It is known that $\beta 1$-integrin can exist in three states: an inactive bent state, a primed extended closed state, and an active extended open state [17, 18]. At present it is unknown whether the consumption of sphingomyelin, the generation of ceramide or a metabolite mediate activation of $\beta 1$-integrin, although previous studies with $P$. aeruginosa suggested that primarily ceramide triggers $\beta 1$-integrin activation [8]. In our studies, we used 9EG7 antibodies to neutralize active $\beta 1$-integrin $[8,18]$. The 9EG7 antibody recognizes the activated extended closed or open state form of $\beta 1$-integrin, which binds to the integrin-epidermal growth factor domain 2 (I-EGF2) within the $\beta 1$-integrin molecule 


\section{Cellular Physiology Cell Physiol Biochem 2018;51:1815-1829

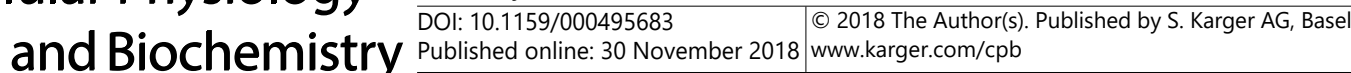 \\ Wu et al.: BCG Induces Granuloma via Neutral Sphingomyelinase and $\beta 1$-Integrin}

[19]. Treatment with 9EG7 antibodies therefore stabilizes its activation state, whereupon it is endocytosed, and its function neutralized.

The results of our studies of immunoprecipitation with 9EG7 antibodies indicate that BCG infection induces a conformational change of $\beta 1$-integrin from the inactivated to the activated form and that this conformational change is strictly dependent on Nsm2 activation. Our findings are consistent with studies from the Karakashian group reporting that the stimulation of JNK phosphorylation is dependent on Nsm2/ceramide-activated protein phosphatase in primary hepatocytes [20].

The presented in vivo examinations did not show classical granuloma, since the granuloma we detected neither contained a tightly organized layer of cells surrounding the center of granuloma nor a necrotic center. This difference might be caused by the reduced pathogenicity of BCG compared to Mycobacterium tuberculosis, but also because the structure of granuloma seems to differ between mice and humans with a lack of necrotic centers even in TB-induced granulomas in mice [21, 22]. However, we found lipid droplet accumulation in Wt granuloma, which was almost absent in $\mathrm{Nsm} 2^{+/-}$mice or in Wt mice after treatment with anti- $\beta 1$-integrin antibodies. It is assumed, that lipid droplets in mycobacterial induced granuloma serve as sole carbon sources, which are essential for mycobacterial survival and growth within granuloma $[23,24]$. The observed lipid droplets seem to be quite similar to those found in nascent granulomas detectable in the lungs of humans with TB [25]. Previous studies suggest that BCG infection in macrophages increases the formation of lipid droplets [26]. In addition, ceramide levels have been reported to be increased in mycobacteria-induced necrotic lung granulomas [25]. More recently, Senkal et al. identified a pathway whereby ceramide is converted to acylceramides for storage in lipid droplets [27]. Here we report that Nsm2 is rapidly activated after BCG infection in Wt macrophages and that this activation results in the generation of ceramide at the inner leaflet of the plasma membrane via hydrolyzing sphingomyelin. This finding raises the possibility that ceramide may be a resource for lipid droplets in BCG infection and that these droplets could be further metabolized by bacteria [28].

\section{Conclusion}

In summary, our findings demonstrate that Nsm2-mediated activation of $\beta 1$-integrin plays a central role in granuloma formation and elimination of BCG in vivo. The interruption of granuloma formation and the protection against mycobacteria infection in mice by heterozygosity of the Nsm2 and neutralizing anti- $\beta 1$-integrin antibodies, suggests that existing therapies may be enhanced by modulating the generation of ceramide by inhibition of the Nsm2. Mice heterozygous for Nsm2 do not have an obvious phenotype and, thus, it can be assumed that drugs that inhibit Nsm2 in humans without complete inhibition will be without side effects and, thus, might be potential drugs to treat tuberculosis.

\section{Acknowledgements}

We thank Simone Keitsch and Melanie Kramer for excellent technical support. The study was supported by DFG grants GR $1697 / 2-2$ to GR and GU 335/30-2 to EG. The authors declare that they have no competing financial interests.

\section{Disclosure Statement}

The authors declare no conflicts of interest. 


\section{Cellular Physiology Cell Physiol Biochem 2018;51:1815-1829 \begin{tabular}{l|l|l} 
and Biol: $10.1159 / 000495683$ & 2018 The Author(s). Published by S. Karger AG, Basel
\end{tabular}

\section{References}

1 WHO. Global tuberculosis report 2017. http://www.who.int/tb/publications/global_report/en/.

2 Gulbins E, Dreschers S, Wilker B, Grassmé H: Ceramide, membrane rafts and infections. J Mol Med (Berl) 2004;82:357-363.

-3 Shamseddine A, Airola MV, Hannun YA: Roles and regulation of neutral sphingomyelinase-2 in cellular and pathological processes. Adv Biol Regul 2015;57:24-41.

4 Faulstich M, Hagen F, Avota E, Kozjak-Pavlovic V, Winkler AC, Xian Y, Schneider-Schaulies S, Rudel T: Neutral sphingomyelinase 2 is a key factor for PorB-dependent invasion of Neisseria gonorrhoeae. Cell Microbiol 2015;17:241-253.

5 Li C., Peng H, Japtok L, Seitz A, Riehle A, Wilker B, Soddemann M, Kleuser B, Edwards M, Lammas D, Zhang Y, Gulbins E, Grassme H: Inhibition of neutral sphingomyelinase protects mice against systemic tuberculosis. Front Biosci (Elite Ed) 2016;8:311-325.

6 Zhang Y, Li X, Carpinteiro A, Gulbins E: Acid sphingomyelinase amplifies redox signaling in Pseudomonas aeruginosa-induced macrophage apoptosis. J Immunol 2008;181:4247-4254.

7 Fazal N, Lammas DA, Raykundalia C, Bartlett R, Kumararatne DS: Effect of blocking TNF-alpha on intracellular BCG (Bacillus Calmette Guerin) growth in human monocyte-derived macrophages. FEMS Microbiol Immunol 1992;5:337-345.

8 Grassmé H, Henry B, Ziobro R, Becker KA, Riethmuller J, Gardner A, Seitz AP, Steinmann J, Lang S, Ward C, Schuchman EH, Caldwell CC, Kamler M, Edwards MJ, Brodlie M, Gulbins E: beta1-Integrin accumulates in cystic fibrosis luminal airway epithelial membranes and decreases sphingosine, promoting bacterial infections. Cell Host Microbe 2017;21:707-718.

9 Puissegur MP, Lay G, Gilleron M, Botella L, Nigou J, Marrakchi H, Mari B, Duteyrat JL, Guerardel Y, Kremer L, Barbry P, Puzo G, Altare F: Mycobacterial lipomannan induces granuloma macrophage fusion via a TLR2dependent, ADAM9- and 1 integrin-mediated pathway. J Immunol 2007;178:3161-3169.

$>10$ Allen WE, Jones GE, Pollard JW, Ridley AJ: Rho, Rac and Cdc42 regulate actin organization and cell adhesion in macrophages. J Cell Sci 1997;110:707-720.

-11 Alrutz MA, Srivastava A, Wong KW, D’Souza-Schorey C, Tang M, Ch'Ng LE, Snapper SB, Isberg RR: Efficient uptake of Yersinia pseudotuberculosis via integrin receptors involves a Rac1-Arp 2/3 pathway that bypasses N-WASP function. Mol Microbiol 2001;42:689-703.

12 Barisch C, Soldati T: Breaking fat! How mycobacteria and other intracellular pathogens manipulate host lipid droplets. Biochimie 2017;141:54-61.

13 Calderwood DA, Shattil SJ, Ginsberg MH: Integrins and actin filaments: reciprocal regulation of cell adhesion and signaling. J Biol Chem 2000;275:22607-22610.

14 Weber GF, Bjerke MA, DeSimone DW: Integrins and cadherins join forces to form adhesive networks. J Cell Sci 2011;124:1183-1193.

15 Bermudez LE, Parker A, Goodman JR: Growth within macrophages increases the efficiency of Mycobacterium avium in invading other macrophages by a complement receptor-independent pathway. Infect Immun 1997;65:1916-1925.

16 McNally AK, Anderson JM: $\beta 1$ and $\beta 2$ Integrins mediate adhesion during macrophage fusion and multinucleated foreign body giant cell formation. Am Journal Pathol 2002;160:621-630.

17 Luo BH, Carman CV, Springer TA: Structural basis of integrin regulation and signaling. Annu Rev Immunol 2007;25:619-647.

$>18$ Carpinteiro A, Becker KA, Japtok L, Hessler G, Keitsch S, Pozgajova M, Schmid KW, Adams C, Muller S, Kleuser B, Edwards MJ, Grassme H, Helfrich I, Gulbins E: Regulation of hematogenous tumor metastasis by acid sphingomyelinase. EMBO Mol Med 2015;7:714-734.

19 Su Y, Xia W, Li J, Walz T, Humphries MJ, Vestweber D, Cabanas C, Lu C, Springer TA: Relating conformation to function in integrin alpha5beta1. Proc Natl Acad Sci USA 2016;113:3872-3881.

20 Karakashian AA, Giltiay NV, Smith GM, Nikolova-Karakashian MN: Expression of neutral sphingomyelinase-2 (NSMase-2) in primary rat hepatocytes modulates IL-beta-induced JNK activation. FASEB J 2004;18:968-970.

-21 Guirado E, Schlesinger LS: Modeling the Mycobacterium tuberculosis granuloma - the critical battlefield in host immunity and disease. Front Immunol 2013;4:98. 


\section{Cellular Physiology Cell Physiol Biochem 2018;51:1815-1829

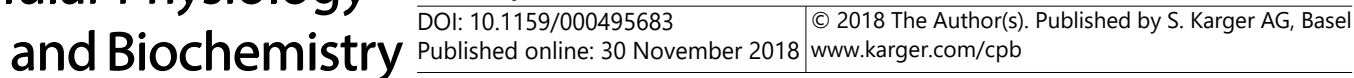

-22 Rhoades ER, Frank AA, Orme IM: Progression of chronic pulmonary tuberculosis in mice aerogenically infected with virulent Mycobacterium tuberculosis. Tuber Lung Dis 1997;78:57-66.

23 Peyron P, Vaubourgeix J, Poquet Y, Levillain F,Botanch C, Bardou F, Daffe M, Emile JF, Marchou F, Cardona PJ, de Chastellier D, Altare F: Foamy macrophages from tuberculous patients' granulomas constitute a nutrient-rich reservoir for M. tuberculosis persistence. PLoS Pathog 2008;4:e1000204.

-24 Russell DG, Vanderven BC, Glennie S, Mwandumba H, Heyderman RS: The macrophage marches on its phagosome: dynamic assays of phagosome function. Nat Rev Immunol 2009;9:594-600.

-25 Kim M.J, Wainwright HC, Locketz M, Bekker LG, Walther GB, Dittrich C, Visser A, Wang W, Hsu FF, Wiehart U, Tsenova L, Kaplan G, Russell GF: Caseation of human tuberculosis granulomas correlates with elevated host lipid metabolism. EMBO Mol Med 2010;2:258-274.

-26 D’Avila H, Melo RC, Parreira GG, Werneck-Barroso E, Castro-Faria-Neto HC, Bozza PT: Mycobacterium bovis Bacillus Calmette-Guerin induces TLR2-mediated formation of lipid bodies: intracellular domains for eicosanoid synthesis in vivo. J Immunol 2006;176:3087-3097.

-27 Senkal CE, Salama MF, Snider AJ, Allopenna JJ, Rana NA, Koller A, Hannun YA, Obeid LM: Ceramide is metabolized to acylceramide and stored in lipid droplets. Cell Metab 2017;25:686-697.

28 Airola MV, Shanbhogue P, Shamseddine AA, Guja KE, Senkal CE, Maini R, Bartke N, Wu BX, Obeid LM, GarciaDiaz M, Hannun YA: Structure of human nSMase2 reveals an interdomain allosteric activation mechanism for ceramide generation. Proc Natl Acad Sci USA 2017;114:5549-E5558. 